\title{
Soluble mesothelin-related peptides levels in patients with malignant mesothelioma
}

\author{
Alenka Franko ${ }^{\mathrm{a}}$, Vita Dolzan ${ }^{\mathrm{b}}$, Viljem Kovac ${ }^{\mathrm{c}}$, Niko Arneric $^{\mathrm{a}}$ and Metoda Dodic-Fikfak ${ }^{\mathrm{a}, *}$ \\ ${ }^{a}$ Clinical Institute of Occupational Medicine, University Medical Center Ljubljana, Poljanski nasip 58, Ljubljana, \\ Slovenia \\ ${ }^{\mathrm{b}}$ Pharmacogenetics Laboratory, Institute of Biochemistry, Faculty of Medicine, University of Ljubljana, Vrazov trg \\ 2, Ljubljana, Slovenia \\ ${ }^{\mathrm{c}}$ Institute of Oncology Ljubljana, Zaloska cesta 2, Ljubljana, Slovenia
}

\begin{abstract}
Soluble mesothelin-related peptides (SMRP) are a potential tumor marker for malignant mesothelioma. The aim of this study was to determine the differences in SMRP levels in patients with malignant mesothelioma before treatment and in various responses to treatment and to investigate whether SMRP level could be useful in evaluating tumor response to treatment. The study included patients with malignant mesothelioma treated at the Institute of Oncology Ljubljana between March 2007 and December 2009. Blood samples were collected before treatment and/or in various responses to treatment. SMRP levels were determined using ELISA assay based upon a combination of two monoclonal antibodies. Mann-Whitney test was used to determine the differences in SMRP levels in various responses to treatment.

Median SMRP was $2.80 \mathrm{nmol} / \mathrm{L}$ (range 0.00-34.80) before treatment, $0.00 \mathrm{nmol} / \mathrm{L}$ (range 0.00-0.00) in complete response, $0.48 \mathrm{nmol} / \mathrm{L}$ (range $0.00-4.40$ ) in partial response, $1.65 \mathrm{nmol} / \mathrm{L}$ (range $0.00-20.71$ ) in stable disease and $7.15 \mathrm{nmol} / \mathrm{L}$ (range 0.44-31.56) in progressive disease. Pre-treatment SMRP levels were significantly higher than in stable disease, partial response and complete response ( $p=0.006$ ), as were SMRP levels in progressive disease compared to stable disease, partial response and complete response $(p<0.001)$.

Our findings suggest that SMRP may be a useful tumor marker for detecting the progression of malignant mesothelioma and evaluating tumor response to treatment.
\end{abstract}

Keywords: Malignant mesothelioma, Soluble Mesothelin-Related Peptides (SMRP), Tumor marker, Tumor response to treatment, Asbestos exposure

\section{Introduction}

Malignant mesothelioma is a highly aggressive tumor of the serosal surfaces [1]. It most commonly occurs in the pleura, but it may also arise in the peritoneum, pericardium, and tunica vaginalis $[1,2]$.

The major cause associated with the development of this disease is asbestos [1,3]. The association be-

\footnotetext{
*Corresponding author: Dr. Metoda Dodic-Fikfak, MD, Assist. Prof., Clinical Institute of Occupational Medicine, University Medical Center, Poljanski nasip 58, 1000 Ljubljana, Slovenia. Tel.: +386 1522 2101; Fax: +386 1522 2478; E-mail: metoda.dodic-fikfak@ guest.arnes.si.
}

tween malignant mesothelioma and occupational [4-7] as well as environmental [8-13] asbestos exposure has been confirmed in many epidemiological studies. The latency period between exposure to asbestos and the onset of malignant mesothelioma is long and can range from 15 to 60 years $[14,15]$.

An accurate and rapid diagnosis of malignant mesothelioma is important for therapeutic reasons [1]. However, this aggressive cancer remains difficult to diagnose in the early phases of the disease. Therefore, potential serum markers that could facilitate an early diagnosis and help to evaluate response to treatment have been extensively investigated; one of these is mesothelin [1,16-22]. Mesothelin is a $40-\mathrm{kDa}$ glycoprotein at- 
tached to the cell surface that is thought to have a role in cell adhesion and possibly in cell-to-cell signaling [19, 20,23-28]. It exists in a variety of forms that can be detected in serum by using monoclonal antibody techniques in the form of soluble mesothelin-related peptides (SMRP) [27]. Mesothelin is highly expressed in malignant mesothelioma [10]. Chang et al. [23] and Ordonez et al. [29] detected mesothelin in all epithelial mesotheliomas and in the epithelial component of biphasic mesothelioma, but their research indicated that it was not expressed in sarcomatous mesotheliomas [23, 29]. Several other studies reported higher levels of SMRP in patients with malignant mesothelioma and suggested that SMRP may be a useful marker for the diagnosis of malignant mesothelioma and in monitoring the disease progression [1,16-21,30]. However, according to the available literature, there have been very few studies that investigated SMRP levels in malignant mesothelioma in relation to varying responses to treatment [30-35]. We thus decided to study the differences in serum SMRP levels before treatment and especially in various responses to treatment (complete response, partial response, stable disease and progressive disease) and to investigate if SMRP level could be useful in evaluating tumor response to treatment.

\section{Patients and methods}

The cross sectional prospective study included all (i.e. 78) patients with malignant mesothelioma treated at the Institute of Oncology Ljubljana from March 2007 to December 2009. Patients with malignant pleural mesothelioma were diagnosed by thoracoscopy and patients with malignant peritoneal mesothelioma by laparoscopy. The diagnosis was confirmed by a histopathological examination performed by a pathologist skilled in diagnosis of this disease. Based on histopathology, the malignant mesotheliomas were classified into the epitheloid, biphasic, and sarcomatoid subtypes. To verify histopathology, the immunohistochemistry methods were used (Cytokeratin 5/6 [CK5/6], Epithelial Membrane Antigen [EMA], Calretinin, Vimentin, Wilms tumor gene-1 [WT1], CD15, Ber-EP4, B72.3, MOC-31, actin, desmin, S-100, Carcinoembryonic Antigen [CEA], thyroid transcriptor factor1 [TTF-1]).

Each patient with pleural mesothelioma was staged with CT scan using the Union for International Cancer Control (UICC) tumor node metastasis (TNM) system [36]. The presence of metastatic disease was evalu- ated for all the patients with pleural malignant mesothelioma. The survival time was determined for all the patients and was defined as time from diagnosis to death.

Most of the patients were treated with 4 to 9 cycles of chemotherapy consisting of low dose gemcitabine in prolonged infusion and cisplatin, or pemetrexed and cisplatin [37-40]. Extrapleural pleuropneumonectomy was performed in one patient with pleural malignant mesothelioma before chemotherapy and in four patients after chemotherapy, and peritonectomy was carried out in two patients with peritoneal malignant mesothelioma before chemotherapy and in three patients after chemotherapy. Four patients received best supportive care only. Among patients with pleural malignant mesothelioma, twenty-nine were treated with second-line chemotherapy and two of them received palliative radiotherapy. The treatment in more details is presented in Fig. 1.

The modified Response Evaluation Criteria in Solid Tumors (RECIST) were used to evaluate tumor response to treatment [41]. The evaluation was performed after the third (initial evaluation response) and the sixth (confirmation of initial response) chemotherapy cycle. Tumor thickness perpendicular to the chest wall or mediastinum was measured in two positions at the three separate levels on thoracic CT scans. The sum of six measurements defined a pleural unidimensional measure. Its reduction of at least $30 \%$ was considered as partial response and an increase of $20 \%$ over the nadir measurement as progressive disease [41].

Data on patients' smoking habits, including the duration of smoking and the total pack-years of smoking, were obtained for all the patients during an interview using a standardized questionnaire [42,43].

Occupational and/or environmental asbestos exposure was determined for all patients. The data regarding occupational asbestos exposure were collected for a group of 17 workers who were exposed to asbestos at an asbestos-cement factory by calculating the cumulative asbestos exposure in fibers $/ \mathrm{cm}^{3}$-years; information regarding the duration and intensity of exposure was available from a previous study [42]. The cumulative asbestos exposure data were divided into five categories (no exposure, very low exposure, low exposure, medium exposure and high exposure). There were no available measurements for the rest of the subjects regarding the intensity of exposure to asbestos, so a thorough work history was obtained with special attention given to exposure time. These workers' histories were used to compare their occupational exposures with the known exposure intensities from the asbestos- 


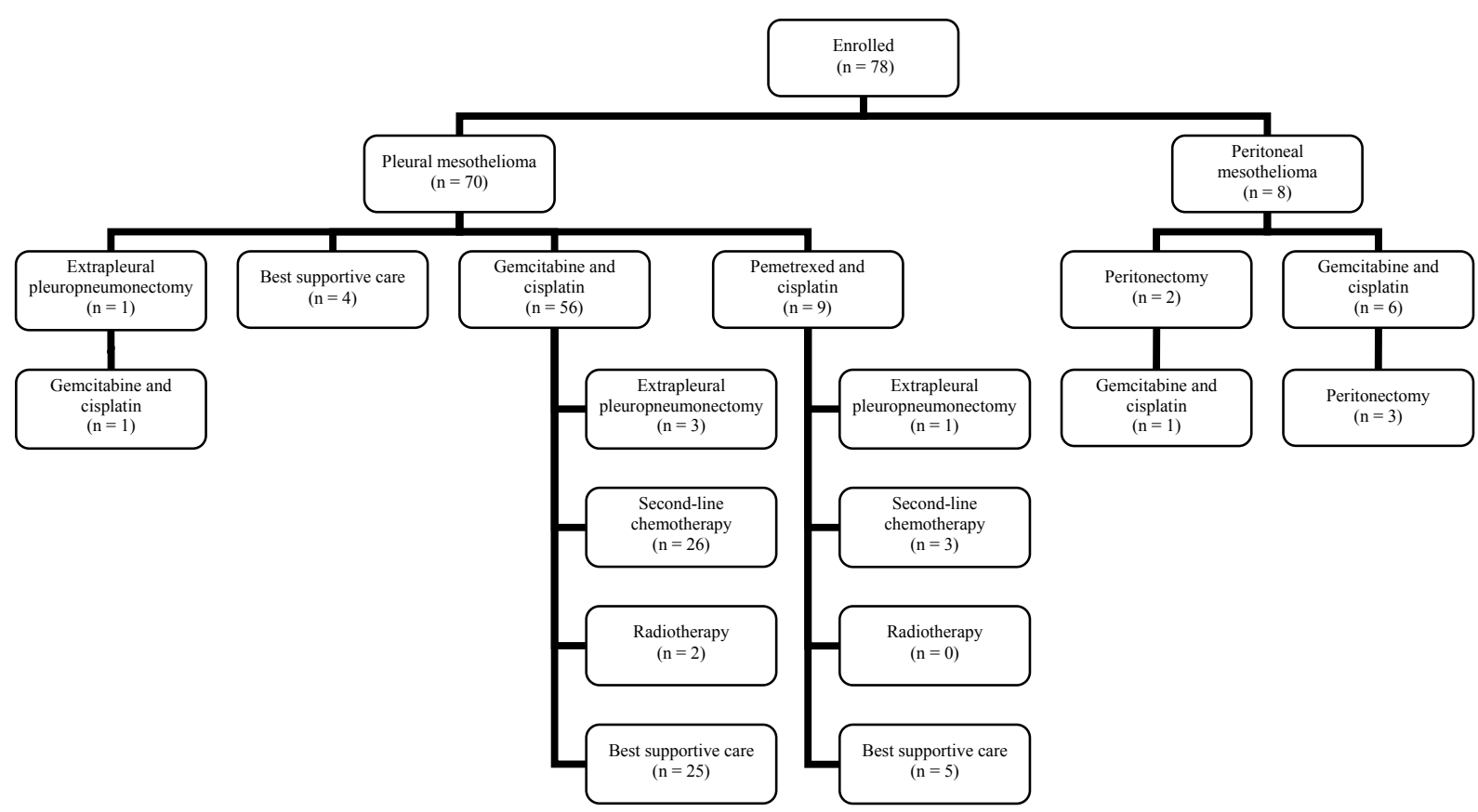

Fig. 1. Treatment of patients with malignant mesothelioma enrolled in the study.

cement factory group. That is, the exposure intensities from the asbestos-cement factory were used as a surrogate for those subjects on whom no exposure intensity information was available. This yielded five categories, containing time of exposure multiplied by category of exposure. Cumulative asbestos exposure for all patients was then grouped into the following four semi-quantitative categories: no exposure, low exposure, medium exposure and high exposure. Two categories (very low and low) from the first group of 17 workers were merged into just one category: low. Environmental asbestos exposure was determined using questions regarding residential exposure: a residence near a source of asbestos exposure; the use of asbestos products or materials indoors (asbestos-cement pipes, linoleum and fire-proof sheets near heat sources, such as cookers); the use of asbestos-cement materials in close proximity to the house (e.g., garden or courtyard); removal or other types of handling of asbestoscontaining corrugated sheets by owners of the houses themselves; and living with family members occupationally exposed to asbestos. Using the same method already described for occupational exposure, all environmental asbestos exposures were classified into the low exposure category.

The latency period defined as the time between the first asbestos exposure and the diagnosis of malignant mesothelioma was also calculated for each patient.
Blood samples for the present study were collected from patients before the treatment (before the 1st cycle of chemotherapy or surgery) and/or after the treatment (after the third and/or the sixth cycle of chemotherapy or surgical procedure) and/or at the progress of the disease. Altogether, 134 blood samples from 78 patients were collected in different periods of treatment and disease. For 32 patients, blood samples were collected both before and after treatment and/or at the progress of the disease, for 5 only before the treatment, while for 41 patients who entered the study during or after treatment the blood samples were collected only after treatment and/or at the progress of the disease. The range of samples taken per patient was 1 to 4 . One blood sample was collected in 43 patients, two blood samples in 17 patients, three blood samples in 15 patients and four blood samples in 3 patients.

Serum was prepared immediately after blood sampling and stored in aliquots frozen at $-30^{\circ} \mathrm{C}$ until the SMRP assay was performed. Serum SMRP levels were determined by sandwich ELISA assay (Mezomark ${ }^{\mathrm{TM}}$ ) using two monoclonal antibodies (4H3 and OV569) and following the manufacturer's protocol (Fujirebio Europe BV, Breda, The Netherlands). According to the previously published literature [44] and manufacturer's data (Mezomark, Fujirebio Diagnostics), we considered $1.50 \mathrm{nmol} / \mathrm{L}$ as a cut-off value and divided SMRP into two categories: $<1.50 \mathrm{nmol} / \mathrm{L}$ and $\geqslant 1.50 \mathrm{nmol} / \mathrm{L}$. 
Table 1

Patients with malignant mesothelioma by gender, age, smoking and asbestos exposure

\begin{tabular}{lc}
\hline & $\mathrm{N}(\%)$ \\
\hline All & 78 \\
Gender & \\
Female & $57(73)$ \\
Male & $21(27)$ \\
Age & Mean (SD) \\
All (years) & $64.48(10.68)$ \\
Smoking & \\
& $\mathrm{N}(\%)$ \\
Never smokers & $43(55.1)$ \\
Ever smokers & $35(44.9)$ \\
& $\mathrm{Mean}(\mathrm{SD})$ \\
Cigarettes per day & $16.29(6.50)$ \\
Years of smoking & $22.63(15.07)$ \\
Pack-years & $19.30(13.72)$ \\
Exposure & \\
Categories & $\mathrm{N}(\%)$ \\
low & $24(30.8)$ \\
median & $21(26.9)$ \\
high & $22(28.2)$ \\
no exposure & $11(14.1)$ \\
& Mean (SD) \\
Duration of exposure (month) & $141.32(149.79)$ \\
\hline
\end{tabular}

Because SMRP levels are also known to increase as a result of renal failure [45], serum creatinine levels were determined simultaneously.

Standard descriptive statistics were used to describe each variable. Considering that the number of enrolled patients and collected blood samples in different phases of the disease was too small for comparing changes within patients, the differences in SMRP levels in various responses to treatment were analyzed across patients by the non-parametric Mann-Whitney (U) test. The correlation between survival and SMRP levels was calculated using Pearson's correlation coefficient.

The study was approved by the Slovenian Ethics Committee for Research in Medicine and was carried out according to the Helsinki Declaration. All the patients gave informed consent to participate in the study.

\section{Results}

The patient characteristics of all 78 patients including gender, age, smoking and asbestos exposure are presented in Table 1 and the patient clinical characteristics in Table 2. At the time of censoring, 8 patients were alive and the mean survival for the overall group was 23 months (Table 2).

The analysis of SMRP levels in 37 patients before treatment showed that levels for the epitheloid subtype were significantly higher compared to the combined
Table 2

Clinical characteristics of patients with malignant mesothelioma

\begin{tabular}{lc}
\hline & $\mathrm{N}(\%)$ \\
\hline Location of disease & $70(89.7)$ \\
Pleura & $8(10.3)$ \\
Peritoneum & \\
Histopathology & $64(82.0)$ \\
Epitheloid & $7(9.0)$ \\
Biphasic & $7(9.0)$ \\
Sarcomatoid & \\
Tumor stage & $5(6.4)$ \\
I & $17(21.8)$ \\
II & $28(35.9)$ \\
III & $20(25.6)$ \\
IV & $8(10.3)$ \\
Not determined (peritoneum) & Month (range) \\
& $23.15(2.83-86.10)$ \\
Mean survival & Years (range) \\
Mean latency period & $38.34(14-56)$ \\
\hline
\end{tabular}

biphasic and sarcomatoid subtypes ( $U=75.00, p=$ 0.020) (Table 3). The SMRP levels before treatment at different tumor stages and according to the presence of metastatic disease in 33 patients with pleural malignant mesothelioma are shown in Table 3. The highest median SMRP level was detected in stage IV (Table 3). Nevertheless, no significant difference in SMRP levels was observed between different tumor stages, except for a borderline significant difference between SMRP levels in stage III and stage IV $(U=34.00, p=0.07)$ (Table 3). There was no significant difference in SMRP levels before treatment in patients with and without evidence of metastatic disease $(U=53.00, p=0.09)$, however, the median SMRP level was higher in patients with metastatic disease (Table 3). No correlation was found between SMRP levels before treatment and survival $(r=0.028 ; p=0.87)$.

The results of the descriptive statistics for SMRP levels before treatment and/or in different responses to treatment for all 78 malignant mesothelioma patients are shown in Table 4. Considering the SMRP cutoff level of $1.50 \mathrm{nmol} / \mathrm{L}, 23(62.2 \%)$ samples were above that level before treatment, no samples were in complete response, $6(46.2 \%)$ samples in partial response, $18(51.4 \%)$ samples in stable disease and 41 (91.1\%) samples in progressive disease (Fig. 2).

SMRP levels before treatment were significantly higher than the levels in complete response $(U=8.00$, $p=0.001)$ and partial response $(U=135.00, p=$ 0.009), while a borderline significant difference was observed between levels before treatment and stable disease $(U=519.50, p=0.075)$. Similarly, higher SMRP levels were found in progressive disease com- 
Table 3

SMRP levels (nmol/L) before treatment at different histopathological subtypes, at different tumor stages and according to the presence of metastatic disease in patients with malignant mesothelioma

\begin{tabular}{|c|c|c|c|c|c|c|c|}
\hline Characteristics & Mean & $\mathrm{SD}$ & Median & Range & Inter-quartile & Mann-Whitney (U) test & $p$ value \\
\hline \multicolumn{8}{|l|}{ Subtype } \\
\hline Epitheloid $(N=27)$ & 7.13 & 8.62 & 3.85 & $0.00-34.80$ & $1.17-9.87$ & $75.00^{\mathrm{a}}$ & 0.020 \\
\hline Biphasic $(N=6)$ & 0.91 & 1.72 & 0.099 & $0.00-4.35$ & $0.00-1.76$ & & \\
\hline Sarcomatoid $(N=4)$ & 3.64 & 2.23 & 4.02 & $0.88-5.64$ & $1.36-5.54$ & & \\
\hline \multicolumn{8}{|l|}{ Tumor stage ${ }^{b}$} \\
\hline \multicolumn{8}{|l|}{$\mathrm{I}^{\mathrm{c}}(N=1)$} \\
\hline $\mathrm{II}(N=8)$ & 3.44 & 3.74 & 2.32 & $0.00-9.84$ & $0.30-7.20$ & $18.00^{\mathrm{d}}$ & 0.16 \\
\hline $\mathrm{III}(N=16)$ & 4.31 & 8.69 & 1.03 & $0.00-34.80$ & $0.28-4.05$ & $34.00^{\mathrm{e}}$ & 0.07 \\
\hline $\mathrm{IV}(N=8)$ & 8.63 & 8.33 & 5.44 & $0.07-24.83$ & $1.96-13.95$ & & \\
\hline \multicolumn{8}{|l|}{ Metastatic disease $^{\mathrm{b}}$} \\
\hline Present $(N=7)$ & 9.12 & 8.88 & 5.64 & $0.07-24.83$ & $1.17-14.01$ & $53.00^{f}$ & 0.09 \\
\hline Not present $(N=26)$ & 4.10 & 7.03 & 1.78 & $0.00-34.80$ & $0.39-4.84$ & & \\
\hline
\end{tabular}

$N=$ number of serum samples;

${ }^{a}$ Mann-Whitney (U) test calculated for epitheloid subtype vs. biphasic + sarcomatoid subtype;

bPleural malignant mesothelioma only;

${ }^{\mathrm{c}}$ Stage I was found only for one patient with SMRP level $4.70 \mathrm{nmol} / \mathrm{L}$;

${ }^{\mathrm{d}}$ Mann-Whitney (U) test calculated for stage II vs. IV;

eMann-Whitney (U) test calculated for stage III vs. IV;

${ }^{\mathrm{f}}$ Mann-Whitney (U) test calculated for metastatic disease present vs. not present.

Table 4

SMRP levels $(\mathrm{nmol} / \mathrm{L})$ before treatment and in different responses to treatment in 78 patients with malignant mesothelioma

\begin{tabular}{lcccccc}
\hline Disease phase & Mean & SD & Median & Range & Inter-quartile & Mann-Whitney $(\mathrm{U})$ test \\
\hline All phases $(N=134)$ & 5.71 & 7.33 & 2.85 & $0.00-34.80$ & $0.89-8.01$ & \\
Before treatment $(N=37)$ & 5.75 & 7.77 & 2.80 & $0.00-34.80$ & $0.71-8.76$ & $662.50^{\mathrm{a}}$ \\
Complete response or after surgery $(N=4)$ & 0.00 & 0.00 & 0.00 & $0.00-0.00$ & $0.00-0.00$ & 0.006 \\
Partial response $(N=13)$ & 1.33 & 1.54 & 0.48 & $0.00-4.40$ & $0.00-2.60$ & \\
Stable disease $(N=35)$ & 2.97 & 4.11 & 1.65 & $0.00-20.71$ & $0.55-3.04$ & \\
Progressive disease $(N=45)$ & 9.67 & 8.34 & 7.15 & $0.44-31.56$ & $3.78-11.86$ & $332.00^{\mathrm{b}}$ \\
\hline
\end{tabular}

$N=$ number of serum samples;

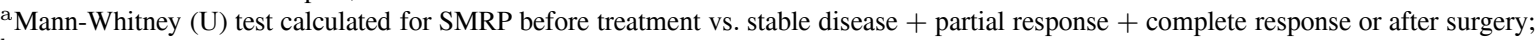

${ }^{\mathrm{b}}$ Mann-Whitney (U) test calculated for SMRP in progressive disease vs. stable disease + partial response + complete response or after surgery.

pared to complete response $(U=0.00, p<0.001)$, partial response $(U=47.00, p<0.001)$ and stable disease $(U=285.00, p<0.001)$. A significant difference in SMRP levels was also observed between complete and partial responses $(U=8.00, p=0.029)$, complete response and stable disease $(U=12.00, p=$ $0.003)$, as well as between levels in progressive disease and before treatment $(U=500.00, p=0.001)$. No significant difference was found between SMRP levels in partial response and stable disease $(U=169, p=$ 0.088). Combining SMRP levels in different phases of the disease showed that the levels were significantly higher before treatment as compared to the combined levels in stable disease, partial response and complete response to treatment $(U=662.50, p=0.006)$. This was also the case in progressive disease compared to the combined levels in stable disease, partial response and complete response to treatment $(U=332.00, p<$ 0.001) (Table 4).
The same analysis was also performed separately for epitheloid mesothelioma. In this case, the MannWhitney test showed a borderline significant difference between SMRP levels in complete and partial responses to treatment $(U=4.00, p=0.071)$, and between levels before treatment and in partial response to treatment ( $U=47.00, p=0.058)$. Other results did not differ importantly from the results observed in all histopathological subtypes together. Serum creatinine levels for all the collected samples were within reference ranges, with a mean value of $83.55 \mu \mathrm{mol} / \mathrm{L}(\mathrm{SD}=23.68)$. They did not exceed the reference value in any of the patients.

\section{Discussion}

The results of this study as well as the findings of other studies published on the subject [4-9,11-13,46,47] 


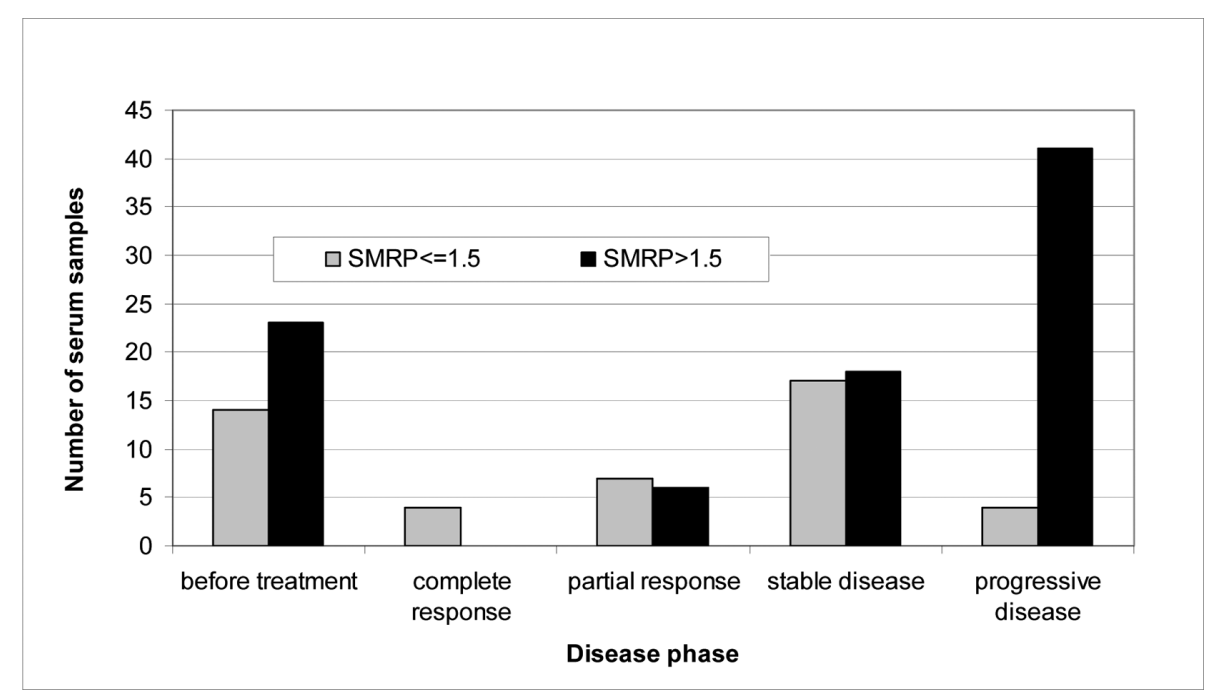

Fig. 2. SMRP levels $<1.5 \mathrm{nmol} / \mathrm{L}$ and $\geqslant 1.5 \mathrm{nmol} / \mathrm{L}$ before treatment and in different responses to treatment in patients with malignant mesothelioma.

have confirmed asbestos exposure in a high percentage of malignant mesothelioma patients.

Several studies have investigated SMRP levels in patients with malignant mesothelioma and have suggested that SMRP may be useful for the detection of this malignoma [1,10,16-19,21,30-33,45]. To our knowledge, however, only a few studies have so far investigated SMRP as a potential tumor marker in relation to varying responses to treatment [30-35].

As expected, the highest median SMRP levels were observed in patients with progressive disease and before treatment. The lowest median levels were found in complete response to treatment or after surgery, where SMRP was zero in all cases, followed by partial response and stable disease. Considering the tumor response criteria [41] and taking into account the suggestion that SMRP levels are directly related to tumor mass $[19,27]$, our results could be biologically plausible. These facts could therefore explain our findings of a significant difference between the serum SMRP levels in progressive disease compared to the levels in stable disease and partial and complete responses to treatment. Similarly, the difference in malignant mesothelioma tumor mass could also clarify the higher median SMRP level before treatment compared to the levels in partial and complete responses to treatment. Our findings are in agreement with Wheatley-Price et al. [33], who found lower postoperative SMRP levels in patients with malignant mesothelioma compared to preoperative levels, and rising SMRP levels in all the patients with radiologic disease progression. Grigoriu et al. [31] also reported increasing serum levels of mesothelin in disease progression and worse outcomes in malignant mesothelioma, whereas stable or decreasing values suggested positive response to treatment. Schneider et al. suggested SMRP as a useful measure in treatment [34], which is similar to the observation of the current study. Recently, Craney et al. [35] reported the potential value of changes in mesothelin levels for monitoring treatment response as well as for prognostication in mesothelioma. Regardless of slightly different results from various studies, it seems that the direction of all studies, including our study, is similar: SMRP appears to be a good tumor marker for evaluating tumor response to treatment.

On the other hand, considering that SMRP level was above $1.5 \mathrm{nmol} / \mathrm{L}$ in only $62.2 \%$ of samples taken before treatment, SMRP seems unlikely to prove significantly useful in screening for malignant mesothelioma, which is in agreement with previous observations [45, 46].

The assessment of treatment response using conventional criteria based upon computed tomography measurements is limited due to the circumferential and axial pattern of growth of malignant mesothelioma [48, 49]. Although the use of fluorodeoxyglucose positron emission tomography (FDG-PET) to assess response to chemotherapy is promising [35,49], new tumor markers, such as SMRP, could also have a particular value in evaluating tumor response to treatment, especially in patients with no measurable disease.

Most of the patients in our study had epitheloid mesothelioma. Among various histopathological subtypes, the highest SMRP levels for blood samples taken 
before treatment were observed in patients with epitheloid mesothelioma as compared to the biphasic and sarcomatoid subtypes. The findings of relatively high values of SMRP in the sacomatoid subgroup although only in 3 cases out of 4 seem to be surprising considering that mesothelin is described not to be expressed in sarcomatoid mesotheliomas [23,29]. However, SMRP levels higher than $1 \mathrm{nmol} / \mathrm{L}$ were also observed in two cases of malignant mesthelioma classified as sarcomatoid in the study of Grigoriu et al. [31] and SMRP levels above $2.5 \mathrm{nmol} / \mathrm{L}$ in several patients with sarcomatoid mesothelioma in the study of Creaney et al. [45]. In any case, the number of patients included in studies has been too small for a reliable conclusion to be drawn.

In this study, no significant difference in SMRP levels before treatment was observed between different tumor stages, although we could not neglect the higher median SMRP level in stage IV. Creaney et al. reported a correlation of baseline serum mesothelin levels with tumor stage; however, they noticed that "some individuals with stage IV had low serum mesothelin" [35]. It should be stressed that there are important interindividual differences in SMRP levels and this could be the reason that comparing SMRP levels between tumor stages among different patients is not significant. The present study found that the median SMRP level before treatment was three times higher in patients with the evidence of metastatic disease compared to those without metastatic disease; however, the difference was not statistically significant. Mesothelin levels did not differ between the patients with and without identified metastases also in the study of Creaney et al. [35]. Contrary to the observation of Creaney et al. [35] that patients with high baseline mesothelin levels have a significantly reduced survival, our analysis did not demonstrate any correlation between patients' survival and baseline SMRP levels. This could be explained by a small number of patients entering the study before treatment and previously mentioned interindividual differences.

Limitations to this study include a small number of patients and the comparison of SMRP levels across patients. We are aware that the comparing changes within patients would be extremely important, especially after we found significant difference in SMRP levels between different responses to treatment across patients. However, we have assessed that the number of enrolled patients and collected blood samples in different phases of the disease is too small for this kind of comparison. Therefore more subjects followed continuously are needed to draw a persuasive conclusion in a future study.

\section{Conclusion}

In conclusion, the study findings suggest that SMRP may be a useful tumor marker for detecting the progression of malignant mesothelioma and in evaluating tumor response to treatment. To confirm these results, more cases are needed to increase the power of the study and to be able to conduct a thorough analytical follow-up study that will also include possible confounders and modifiers of exposure and disease.

Although our results show statistically significant differences between the SMRP levels in patients with a complete response and those in other phases of the disease, it is still an open question whether SMRP is a good marker for a routine evaluation of tumor response to treatment in individual malignant mesothelioma cases.

\section{Acknowledgements}

This research was financially supported by the Ministry of Education, Science and Sport of the Republic of Slovenia (Grants Nos. L3-9129 and P1-0170). We would also like to express our thanks to the staff and especially Barbara Mozina, MSc, director of the biochemistry laboratory at the Institute of Oncology Ljubljana, for their expert help with blood sample collection and handling.

\section{Manufacturer Name}

Serum SMRP levels were determined by sandwich ELISA assay (Mezomark ${ }^{\mathrm{TM}}$ ) following the manufacturer's protocol (Fujirebio Europe BV, Breda, The Netherlands).

\section{References}

[1] B.W. Robinson and R.A. Lake, Advances in malignant mesothelioma, N Engl J Med 353 (2005), 1591-1603.

[2] J.E. King and P.S. Hasleton, The epidemiology and aetiology of malignant mesothelioma, in: Malignant Pleural Mesothelioma, K. O'Byrne and V. Rusch, eds, Oxford University Press, Oxford, New York, 2006, pp. 1-18.

[3] J.C. Wagner, C.A. Sleggs and P. Marchand, Diffuse pleural mesothelioma and asbestos exposure in the North Western Cape Province, Br J Ind Med 17 (1960), 260-271.

[4] A. Agudo, C.A. Gonzalez, M.J. Bleda, J. Ramirez, S. Hernandez, F. Lopez, A. Calleja, R. Panades, D. Turuguet, A. Escolar, M. Beltran and J.E. Gonzalez-Moya, Occupation and risk of malignant pleural mesothelioma: A case-control study in Spain, Am J Ind Med 37 (2000), 159-168. 
[5] D. Howel, A. Gibbs, L. Arblaster, L. Swinburne, M. Schweiger, E. Renvoize, P. Hatton and F. Pooley, Mineral fibre analysis and routes of exposure to asbestos in the development of mesothelioma in an English region, Occup Environ Med 56 (1999), 51-58.

[6] Y. Iwatsubo, J.C. Pairon, C. Boutin, O. Menard, N. Massin, D. Caillaud, E. Orlowski, F. Galateau-Salle, J. Bignon and P. Brochard, Pleural mesothelioma: dose-response relation at low levels of asbestos exposure in a French population-based case-control study, Am J Epidemiol 148 (1998), 133-142.

[7] L. Zellos and D.C. Christiani, Epidemiology, biologic behavior, and natural history of mesothelioma, Thorac Surg Clin $\mathbf{1 4}$ (2004), 469-477, viii.

[8] R.P. Abratt, D.A. Vorobiof and N. White, Asbestos and mesothelioma in South Africa, Lung Cancer 45(Suppl 1) (2004), S3-S6

[9] M. Berry, Mesothelioma incidence and community asbestos exposure, Environ Res 75 (1997), 34-40.

[10] R. Hassan and M. Ho, Mesothelin targeted cancer immunotherapy, Eur J Cancer 44 (2008), 46-53.

[11] C. Magnani, P. Dalmasso, A. Biggeri, C. Ivaldi, D. Mirabelli and $\mathrm{B}$. Terracini, Increased risk of malignant mesothelioma of the pleura after residential or domestic exposure to asbestos: a case-control study in Casale Monferrato, Italy, Environ Health Perspect 109 (2001), 915-919.

[12] M.M. Maule, C. Magnani, P. Dalmasso, D. Mirabelli, F. Merletti and A. Biggeri, Modeling mesothelioma risk associated with environmental asbestos exposure, Environ Health Perspect 115 (2007), 1066-1071.

[13] X.L. Pan, H.W. Day, W. Wang, L.A. Beckett and M.B. Schenker, Residential proximity to naturally occurring asbestos and mesothelioma risk in California, Am J Respir Crit Care Med 172 (2005), 1019-1025.

[14] C. Bianchi, L. Giarelli, G. Grandi, A. Brollo, L. Ramani and C. Zuch, Latency periods in asbestos-related mesothelioma of the pleura, Eur J Cancer Prev 6 (1997), 162-166.

[15] D.M. McElvenny, A.J. Darnton, M.J. Price and J.T. Hodgson, Mesothelioma mortality in Great Britain from 1968 to 2001, Occup Med (Lond) 55 (2005), 79-87.

[16] H.L. Beyer, R.D. Geschwindt, C.L. Glover, L. Tran, I. Hellstrom, K.E. Hellstrom, M.C. Miller, T. Verch, W.J. Allard, H.I. Pass and N.Y. Sardesai, MESOMARK: a potential test for malignant pleural mesothelioma, Clin Chem 53 (2007), $666-672$.

[17] A. Cristaudo, R. Foddis, A. Vivaldi, G. Guglielmi, N. Dipalma, R. Filiberti, M. Neri, M. Ceppi, M. Paganuzzi, G.P. Ivaldi, M. Mencoboni, P.A. Canessa, N. Ambrosino, A. Chella, L. Mutti and R. Puntoni, Clinical significance of serum mesothelin in patients with mesothelioma and lung cancer, Clin Cancer Res 13 (2007), 5076-5081.

[18] B.D. Grigoriu, A. Scherpereel, P. Devos, B. Chahine, M. Letourneux, P. Lebailly, M. Gregoire, H. Porte, M.C. Copin and P. Lassalle, Utility of osteopontin and serum mesothelin in malignant pleural mesothelioma diagnosis and prognosis assessment, Clin Cancer Res 13 (2007), 2928-2935.

[19] B.W. Robinson, J. Creaney, R. Lake, A. Nowak, A.W. Musk, N. de Klerk, P. Winzell, K.E. Hellstrom and I. Hellstrom, Mesothelin-family proteins and diagnosis of mesothelioma, Lancet 362 (2003), 1612-1616.

[20] B.W. Robinson, J. Creaney, R. Lake, A. Nowak, A.W. Musk, N. de Klerk, P. Winzell, K.E. Hellstrom and I. Hellstrom, Soluble mesothelin-related protein-a blood test for mesothelioma, Lung Cancer 49(Suppl 1) (2005), S109-S111.
[21] A. Scherpereel, B. Grigoriu, M. Conti, T. Gey, M. Gregoire, M.C. Copin, P. Devos, B. Chahine, H. Porte and P. Lassalle, Soluble mesothelin-related peptides in the diagnosis of malignant pleural mesothelioma, Am J Respir Crit Care Med 173 (2006), 1155-1160.

[22] J. Creaney, D. Yeoman, A.W. Musk, N. de Klerk, S.J. Skates and B.W. Robinson, Plasma versus serum levels of osteopontin and mesothelin in patients with malignant mesotheliomaWhich is best? Lung Cancer, Mar 11, 2011, [Epub ahead of print].

[23] K. Chang, L.H. Pai, J.K. Batra, I. Pastan and M.C. Willingham, Characterization of the antigen (CAK1) recognized by monoclonal antibody $\mathrm{K} 1$ present on ovarian cancers and normal mesothelium, Cancer Res 52 (1992), 181-186.

[24] K. Chang and I. Pastan, Molecular cloning of mesothelin, a differentiation antigen present on mesothelium, mesotheliomas, and ovarian cancers, Proc Natl Acad Sci U S A 93 (1996), 136-140.

[25] P.S. Chowdhury, J.L. Viner, R. Beers and I. Pastan, Isolation of a high-affinity stable single-chain Fv specific for mesothelin from DNA-immunized mice by phage display and construction of a recombinant immunotoxin with anti-tumor activity, Proc Natl Acad Sci U S A 95 (1998), 669-674.

[26] T. Kojima, M. Oh-eda, K. Hattori, Y. Taniguchi, M. Tamura, N. Ochi and N. Yamaguchi, Molecular cloning and expression of megakaryocyte potentiating factor cDNA, J Biol Chem $\mathbf{2 7 0}$ (1995), 21984-21990.

[27] M.D. Peake, J. Entwisle and S.G. Gray, Malignant pleural mesothelioma: clinical presentation, radiological evaluation and diagnosis., in: Malignant Pleural Mesothelioma, $\mathrm{K}$. O'Byrne and V. Rusch, eds, Oxford University Press, Oxford, New York, 2006, pp. 35-60.

[28] N. Scholler, N. Fu, Y. Yang, Z. Ye, G.E. Goodman, K.E. Hellstrom and I. Hellstrom, Soluble member (s) of the mesothelin/megakaryocyte potentiating factor family are detectable in sera from patients with ovarian carcinoma, Proc Natl Acad Sci U S A 96 (1999), 11531-11536.

[29] N.G. Ordonez, Value of mesothelin immunostaining in the diagnosis of mesothelioma, Mod Pathol 16 (2003), 192-197.

[30] M. Amati, M. Tomasetti, M. Scartozzi, L. Mariotti, R. Alleva, E. Pignotti, B. Borghi, M. Valentino, M. Governa, J. Neuzil and L. Santarelli, Profiling tumor-associated markers for early detection of malignant mesothelioma: an epidemiologic study, Cancer Epidemiol Biomarkers Prev 17 (2008), 163-170.

[31] B.D. Grigoriu, B. Chahine, A. Vachani, T. Gey, M. Conti, D.H. Sterman, G. Marchandise, H. Porte, S.M. Albelda and A. Scherpereel, Kinetics of soluble mesothelin in patients with malignant pleural mesothelioma during treatment, Am J Respir Crit Care Med 179 (2009), 950-954.

[32] K. Tajima, M. Hirama, K. Shiomi, T. Ishiwata, M. Yoshioka, A. Iwase, S. Iwakami, M. Yamazaki, M. Toba, K. Tobino, K. Sugano, M. Ichikawa, Y. Hagiwara, K. Takahashi and O. Hino, ERC/mesothelin as a marker for chemotherapeutic response in patients with mesothelioma, Anticancer Res 28 (2008), 39333936.

[33] P. Wheatley-Price, B. Yang, D. Patsios, D. Patel, C. Ma, W. Xu, N. Leighl, R. Feld, B.C. Cho, B. O'Sullivan, H. Roberts, M.S. Tsao, M. Tammemagi, M. Anraku, Z. Chen, M. de Perrot and G. Liu, Soluble mesothelin-related Peptide and osteopontin as markers of response in malignant mesothelioma, J Clin Oncol 28 (2010), 3316-3322.

[34] J. Schneider, H. Hoffmann, H. Dienemann, F.J. Herth, M. Meister and T. Muley, Diagnostic and prognostic value of soluble mesothelin-related proteins in patients with malignant 
pleural mesothelioma in comparison with benign asbestosis and lung cancer, J Thorac Oncol 3 (2008), 1317-1324.

[35] J. Creaney, R.J. Francis, I.M. Dick, A.W. Musk, B.W. Robinson, M.J. Byrne and A.K. Nowak, Serum soluble mesothelin concentrations in malignant pleural mesothelioma: relationship to tumor volume, clinical stage and changes in tumor burden, Clin Cancer Res 17 (2011), 1181-1189.

[36] UICC International Union Against Cancer, TNM Classification on Malignant Tumours, L. Sobin and C. Wittekind, eds, Sixth edition. New York: Wiley-Liss, 2002.

[37] V. Kovac, M. Zwitter and M. Rajer, Low-dose gemcitabine in prolonged infusion and cisplatin for the treatment of malignant pleural mesothelioma. [Abstract], J Thorac Oncol 6(Suppl) (2011), S1359-S1360.

[38] M. Zwitter, V. Kovac, M. Rajer, M. Vrankar and U. Smrdel, Two schedules of chemotherapy for patients with non-small cell lung cancer in poor performance status: a phase II randomized trial, Anticancer Drugs 21 (2010), 662-668.

[39] A.S. Tsao, R. Mehran and J.A. Roth, Neoadjuvant and intrapleural therapies for malignant pleural mesothelioma, Clin Lung Cancer 10 (2009), 36-41.

[40] N.J. Vogelzang, J.J. Rusthoven, J. Symanowski, C. Denham, E. Kaukel, P. Ruffie, U. Gatzemeier, M. Boyer, S. Emri, C. Manegold, C. Niyikiza and P. Paoletti, Phase III study of pemetrexed in combination with cisplatin versus cisplatin alone in patients with malignant pleural mesothelioma, J Clin Oncol 21 (2003), 2636-2644.

[41] M.J. Byrne and A.K. Nowak, Modified RECIST criteria for assessment of response in malignant pleural mesothelioma, Ann Oncol 15 (2004), 257-260.
[42] M. Dodic Fikfak, D. Kriebel, M.M. Quinn, E.A. Eisen and D.H. Wegman, A case control study of lung cancer and exposure to chrysotile and amphibole at a Slovenian asbestoscement plant, Ann Occup Hyg 51 (2007), 261-268.

[43] B.G. Ferris, Epidemiology Standardization Project (American Thoracic Society), Am Rev Respir Dis 118 (1978), 1-120.

[44] M. Tomasetti and L. Santarelli, Biomarkers for early detection of malignant mesothelioma: diagnostic and therapeutic application, Cancers 2 (2010), 523-548.

[45] J. Creaney, I. van Bruggen, M. Hof, A. Segal, A.W. Musk, N. de Klerk, N. Horick, S.J. Skates and B.W. Robinson, Combined CA125 and mesothelin levels for the diagnosis of malignant mesothelioma, Chest 132 (2007), 1239-1246.

[46] C. Bianchi and T. Bianchi, Malignant pleural mesothelioma in Italy, Indian J Occup Environ Med 13 (2009), 80-83.

[47] J. Hansen, N.H. de Klerk, A.W. Musk and M.S. Hobbs, Environmental exposure to crocidolite and mesothelioma: exposure-response relationships, Am J Respir Crit Care Med 157 (1998), 69-75.

[48] G.L. Ceresoli, A. Chiti, P.A. Zucali, F. Cappuzzo, F. De Vincenzo, R. Cavina, M. Rodari, D. Poretti, F.R. Lutman and A. Santoro, Assessment of tumor response in malignant pleural mesothelioma, Cancer Treat Rev 33 (2007), 533-541.

[49] B.E. Wilcox, R.M. Subramaniam, P.J. Peller, G.L. Aughenbaugh, F.C. Nichols Iii, M.C. Aubry and J.R. Jett, Utility of integrated computed tomography-positron emission tomography for selection of operable malignant pleural mesothelioma, Clin Lung Cancer 10 (2009), 244-248. 


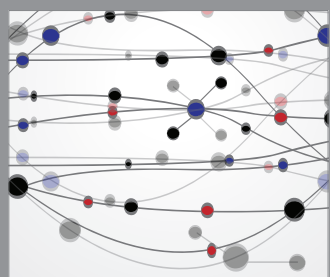

The Scientific World Journal
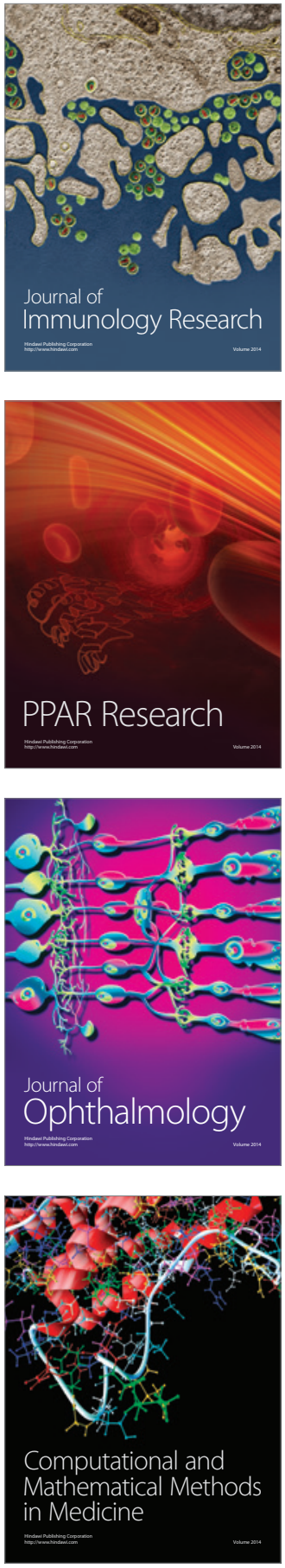

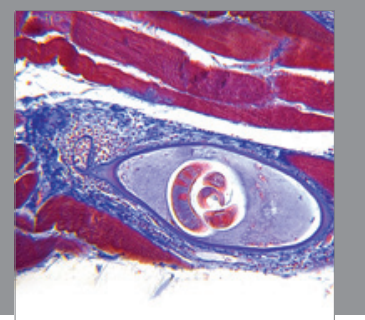

Gastroenterology

Research and Practice
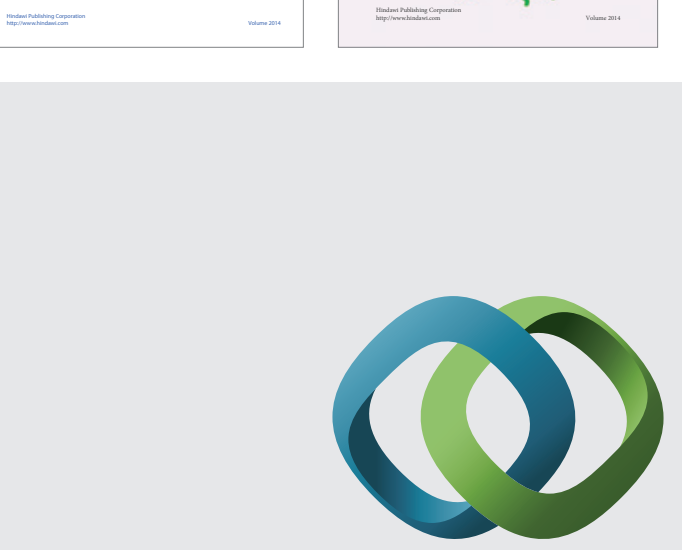

\section{Hindawi}

Submit your manuscripts at

http://www.hindawi.com
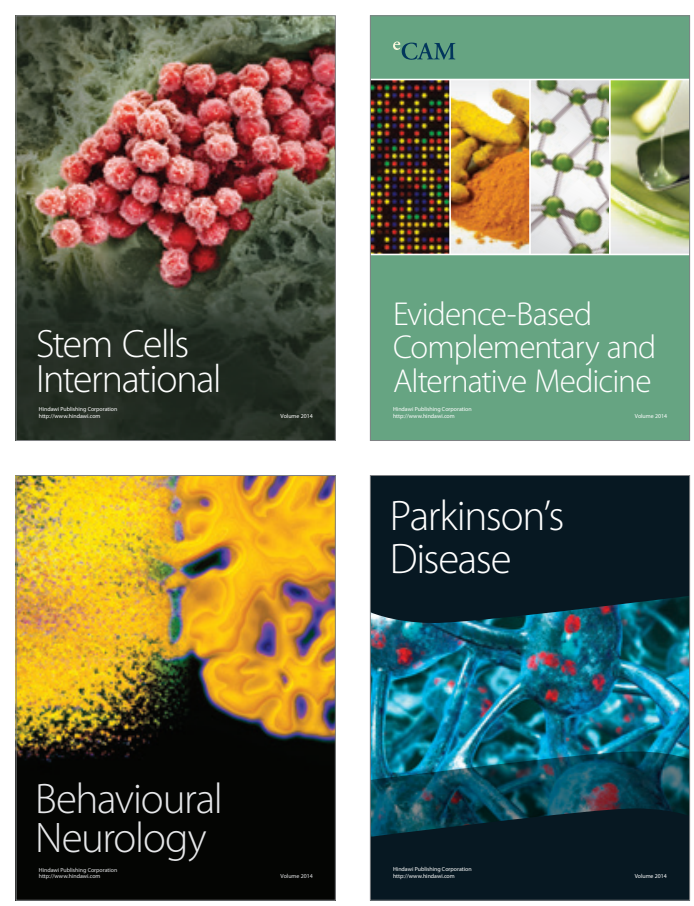

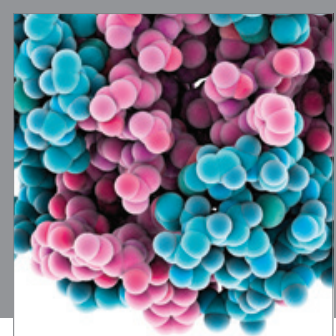

Journal of
Diabetes Research

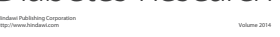

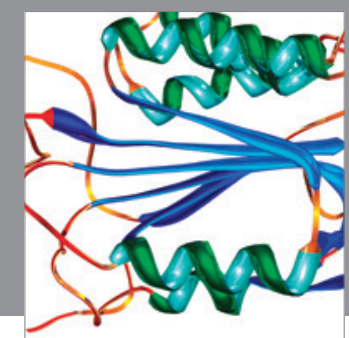

Disease Markers
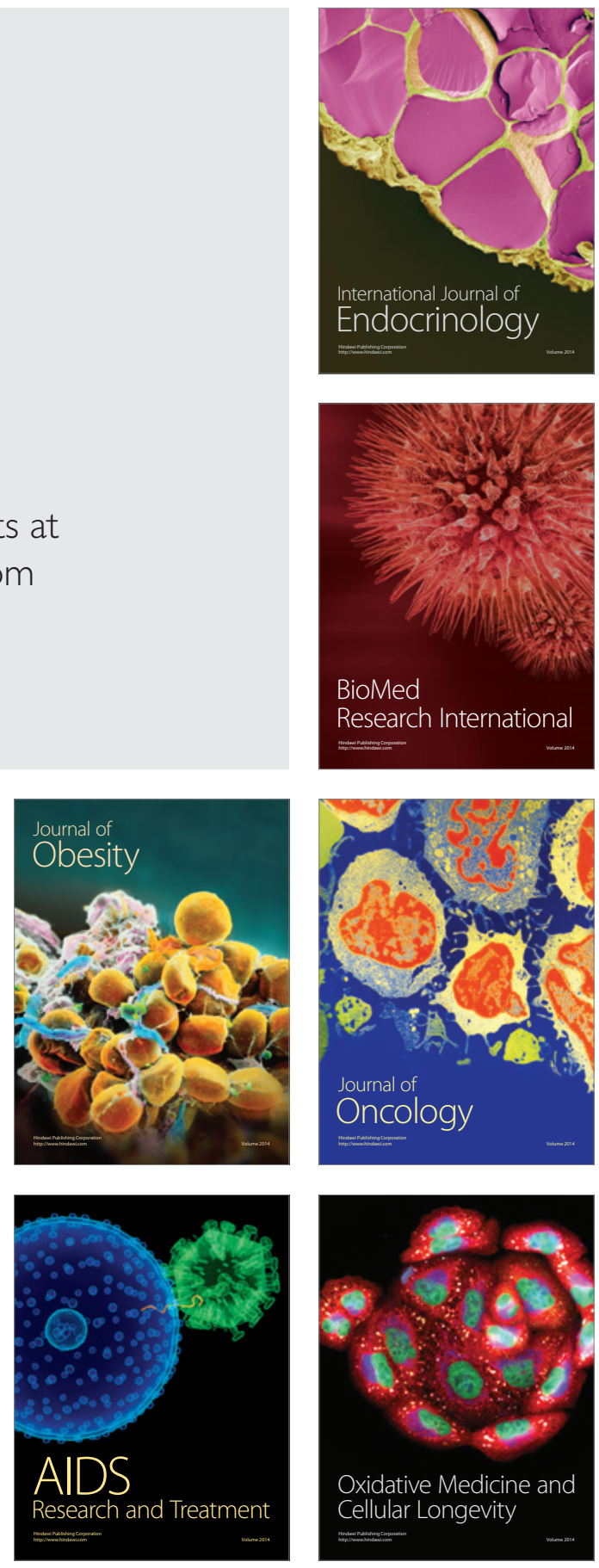